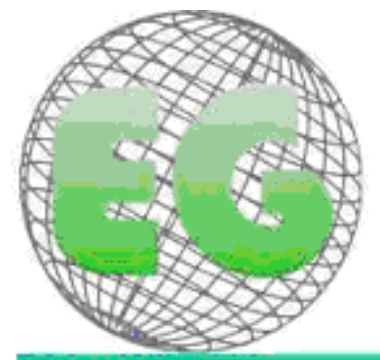

15SN 1696-6*5:

\title{
LA INSERCIÓN DE LAS PLANTAS MEDICINALES EN LA PRÁCTICA DE ENFERMERÍA: UN CRECIENTE DESAFÍO
}

A INSERÇÃO DAS PLANTAS MEDICINAIS ENQUANTO PRÁTICA DA ENFERMAGEM: UM CRESCENTE DESAFIO

\author{
${ }^{*}$ Borges, AM.; ${ }^{* *}$ Ceolin, T.; ${ }^{* * *}$ Barbieri, RL.; ${ }^{* * * H e c k, ~ R M . ~}$ \\ *Enfermera. Mestranda del Programa de Pós-Graduação em Enfermagem da Universidade \\ Federal de Pelotas (UFPel). "*Mestre em Enfermagem da UFPel. "** Dra. en Genética y \\ Biología Molecular. Pesquisadora da Embrapa Clima Temperado. "*** Dra. en Enfermería. \\ Profesora Adjunta del curso de Enfermería de la UFPel. Brasil.
}

\author{
Palabras clave: Salud, Medioambiente, Biodiversidad \\ Palavras chaves: Saúde, Meio ambiente, Biodiversidade \\ Keywords: Health, Environment, Biodiversity.
}

\section{RESUMEN}

Brasil cuenta con un patrimonio genético y cultural de valor equivalente al $20 \%$ de las especies de plantas en el planeta. Esta biodiversidad está siendo utilizada con fines terapéuticos desde los albores de la humanidad, donde el ser humano, a través de las plantas medicinales, comenzó a buscar en la naturaleza la cura de enfermedades y el alivio de los síntomas perjudiciales para su salud. En esta perspectiva, la Organización Mundial de la Salud, reconociendo la necesidad de combinar el potencial herbario de Brasil con la atención primaria de salud, incorporó las plantas medicinales en el ámbito sanitario, estableciendo las políticas gubernamentales enfocadas en la transversalidad de las acciones públicas en salud. En este sentido, se propone una reflexión sobre la aplicación del cuidado a través de la inserción de plantas medicinales como terapia complementaria en la enfermería, desafíos y percepciones. Se observa que uno de los factores que dificultan esta iniciativa es la lentitud en la aprobación de los estudios científicos relacionados con el uso de plantas con fines terapéuticos, unido al escaso número de profesionales de la salud que actúan de forma comprometida y multidisciplinar, convirtiendo el conocimiento popular en saberes científicos con el fin de rescatar el patrimonio cultural valorando el medio ambiente como parte indisociable de la persona. 


\section{RESUMO}

O Brasil possui um patrimônio genético e cultural significativo correspondente a $20 \%$ das espécies vegetais do planeta. Esta biodiversidade está sendo utilizada para fins terapêuticos desde os primórdios da humanidade, onde o ser humano, através da adesão das plantas medicinais, passou a buscar na natureza a cura de enfermidades e o alívio de sintomas prejudiciais à sua saúde. Nesta perspectiva, a Organização Mundial de Saúde, reconhecendo a necessidade de unir o potencial herbário do Brasil à atenção básica em saúde, incorporou as plantas medicinais no âmbito sanitário, estabelecendo, para tanto, diretrizes governamentais com foco na transversalidade das ações públicas em saúde. Sob este aspecto, propõe-se uma reflexão acerca da implementação do cuidado através da inserção das plantas medicinais enquanto terapia complementar no campo da enfermagem, desafios e percepções. Observa-se que um dos fatores que dificultam esta iniciativa é a lentidão na apropriação dos estudos científicos vinculados à utilização das plantas para fins terapêuticos, associado ainda, ao reduzido número de profissionais de saúde que atuam de forma comprometida e multidisciplinar, transformando os conhecimentos populares em saberes científicos, de modo a resgatar a herança cultural valorizando o meio enquanto parte indissociável do indivíduo.

\section{INTRODUCCIÓN}

El proceso histórico de la utilización de las plantas medicinales como fuente terapéutica tuvo su inicio durante la propia evolución humana, cuyas prácticas volcadas al cuidado de la salud estaban orientadas a los conocimientos populares pasados de generación a generación ${ }^{1}$.

De acuerdo con la Organización Mundial de la Salud (OMS), la mayoría de la población de los países en desarrollo recurre a la medicina tradicional como fuente de atención primaria en salud, siendo que la utilización de las plantas medicinales representa en torno al $85 \%$ de las iniciativas. A mediados de 1978, a través de la Declaración de Alma-Ata se evidencia el interés de la OMS en incorporar las plantas en el ámbito sanitario².

En este enfoque, se hace oportuna una reflexión sobre el concepto de plantas medicinales. La OMS afirma que plantas medicinales son especies vegetales con finalidades terapéuticas $^{2}$ que, cultivadas o no, demandan cuidados en su uso, pues pueden ofrecer riesgos a la salud al ser manipuladas de forma equivocada ${ }^{3}$.

En 1988 fue reglamentada en Brasil la implantación de la fitoterapia en el ámbito de la salud, a través de Comisión Interministerial de Planificación y Coordinación (Ciplan). Y en 1996 representantes de la $10^{\underline{a}}$ Conferencia Nacional de Salud propusieron incorporar tal práctica en el Sistema Único de Salud (SUS) ${ }^{4}$.

Tales iniciativas se sumaron a varias otras en el transcurso de los años en Brasil, siendo establecidas directrices gubernamentales con enfoque en la transversalidad de las acciones públicas, económicas y sociales en salud, en búsqueda del uso sostenible de los recursos naturales. Surgió, entonces, la Política Nacional de Plantas Medicinales y Fitoterápicos, en 2006, que reglamentaba la utilización de la terapia complementaria de forma racional y aliada a las prácticas tradicionales de salud ${ }^{2}$.

Esta propuesta contribuyó al progreso de calidad de vida de la población brasileña, así como a la conservación ambiental y al respeto a los principios de seguridad y eficacia en el manejo de las plantas medicinales. 
Con relación al trabajo profesional de enfermería, se percibe que éste enfrenta algunas dificultades en incluir en su praxis diaria la utilización de las terapias complementarias, en especial las plantas medicinales, teniendo en cuenta que sus conocimientos todavía son reducidos y muy próximos al sentido común ${ }^{3}$. De ahí la necesidad de perfeccionamiento de los profesionales de salud, en cuanto a la utilización de las plantas medicinales, posibilitando la ampliación de la oferta del cuidado integral, con focalización interdisciplinaria.

Considerando lo expuesto, se evidencia la necesidad de la enfermería en conocer el contexto en el cual actúa y valorar la herencia cultural, realizando investigaciones a fin de contribuir a que la asistencia sea un campo de interrelaciones entre sujetos activos, que participan del proceso de tener salud y ser saludable.

Se propone una reflexión con relación a la implementación del cuidado a través de la inserción de las plantas medicinales en cuanto terapia complementaria en el campo de la enfermería, sus desafíos y percepciones.

\section{EL AMBIENTE Y EL SER HUMANO, UNA CUESTIÓN DE EDUCACIÓN EN SALUD}

Brasil tiene un amplio patrimonio genético y cultural que corresponde al $20 \%$ de la flora de todo el mundo. Cerca de sesenta mil especies vegetales ya fueron catalogadas y entre ellas están las plantas medicinales, que abarcan recursos orientados a la promoción de la salud y prevención de enfermedades, desde los principios de la humanidad ${ }^{2}$.

La diversidad étnica existente en Brasil ayudó mucho en la construcción de conocimientos populares volcados a la atención de la salud. Tal práctica se debe a la articulación de conocimientos indígenas, africanos y portugueses, pueblos pioneros en el uso de la flora y fauna brasileña ${ }^{4}$. A través del mestizaje de estos primeros habitantes, la diversificación del bagaje cultural sobre el uso de las plantas y sus aspectos medicinales se propagó, desencadenando su uso de forma intuitiva y empírica ${ }^{3}$.

En este proceso, la ideología capitalista fomentó el interés popular en explorar los recursos naturales con la intención de acumular riquezas, ejecutando prácticas depredatorias en las selvas y vegetaciones nativas. La escasez de recursos naturales, asociada a la degradación del medio ambiente, resulta del crecimiento demográfico de los países en desarrollo.

Insertado en esta perspectiva, el individuo, con su concepción de superioridad al ambiente, pasó a explorarlo sin percibir que su supervivencia está directamente relacionada con él.

De esta forma, los impactos de la depredación indebida del reino vegetal, pasaron a convertirse en agresiones también para la salud humana, que sufre en su constitución genética y fenotípica, influencias de acuerdo con la relación existente entre el ser humano y la naturaleza. De ahí la importancia de mantener afinado en este vínculo, el respeto y la responsabilidad, de modo a garantizar la manutención de las riquezas naturales a través del uso sostenible de la biodiversidad existente y ofrecer a la población la calidad de vida constantemente idealizada ${ }^{1-4}$.

Valores enraizados en la cultura contemporánea trajeron, entonces, varias concepciones sobre salud/enfermedad distintas del enfoque de prevención, cura y promoción, volcadas a la filosofía individualista, concebida por el modelo biomédico de enfoque hegemónico clínico, vigente en Brasil. De esta manera, la ascensión social orientó al ser humano a unir el "estar saludable" al status de ser bello, joven delgado y activo; formas que idealizan mucho más el cuerpo y la apariencia física que la mente, manteniéndolos así, distantes de su equilibrio ${ }^{5}$. 
En esta concepción, emerge la necesidad de resignificación de la salud, pues la inserción de las actitudes cosificadas y fragmentadas corroboran al surgimiento de una crisis ecológica inducida por el propio individuo, que con el uso obcecado de la ciencia y de la razón tecnológica, desprecia parte de su existencia: el medio ambiente ${ }^{5}$.

Se entiende que es preciso ecologizar el pensamiento, buscando una reflexión sobre las intervenciones humanas en la sociedad que cualifican el ser y estar en el mundo. Adoptar posturas críticas y con intereses colectivos, que permiten convivir de forma organizada y saludable con el medio. $Y$ esto implica percibir que los fragmentos forman parte de una integralidad, uniéndolos.

En este filosofar, la educación en salud se constituye como proceso que incentiva a la población a adoptar una postura consciente y autónoma en su plan de cuidados ${ }^{6}$. Por eso, percibir, oír y sentir y relacionarse con el ambiente pasa a tener otra visión, pues permite que las generaciones y las culturas se propaguen, con un reducido impacto sobre los recursos naturales.

Disparidades siempre existirán, es preciso que el individuo busque la preservación del lugarl en que sobrevive, delimitando acciones que cuiden del ambiente, haciéndolo saludable, libre de riesgos de morbimortalidades. De esta forma, se constata que no hay jerarquía entre el medio y los seres vivos, mas sí una organización sistémica, en que sufrimos constantes desequilibrios en búsqueda de una estabilidad emocional, social y física que jamás será absoluta. Así, la búsqueda por la supervivencia lleva el individuo a sufrir adaptaciones que inciden en su actuar y pensar, sin embargo, muchas veces, la incoherencia en las actitudes y la falta de conciencia respecto al elemento integrante del medio en que vive, lo lleva a su autodestrucción, lo que deja al mundo enfermo e impaciente por la vida.

En medio de la pluralidad de la ciencia humana, los profesionales de salud precisan estar abiertos para evolucionar y emancipar sus conocimientos, pues su campo de actuación es rico $^{7}$ y propicio para incentivar el autocuidado de forma segura, teniendo en cuenta que la esencia de la enfermería reside en al acto de cuidar y el proceso de aprendizaje es un ejercicio continuado.

Este contexto posibilita a la enfermería buscar la ampliación de sus conocimientos, considerando el saber popular en la realización del cuidado integral al individuo y a su familia, valorando el ámbito social, económico y cultural en el cual está insertado.

\section{LA INSERCIÓN DE LAS PLANTAS MEDICINALES EN CUANTO PRÁCTICA DEL CUIDADO EN SALUD}

Al repensar las prácticas de cuidado respecto a la atención integral a la salud es esencial considerar el contexto cultural, la dimensión de tiempo y espacio en que la población está insertada, proponiendo comprender los recurso disponibles, las acciones en salud incorporadas y la representación social de salud/enfermedad ${ }^{6-8}$.

Así se observa que la adhesión a las plantas medicinales es muy antigua, existe desde los orígenes de la humanidad, cuya participación popular en el cuidado de sí misma, buscaba la cura de enfermedades y el alivio de síntomas perjudiciales para la salud. Hoy, tal propuesta todavía persiste, pues muchas personas recurren a las especies vegetales para fines de tratamiento. En este sentido, la enfermería, a través de su percepción holística en salud, agrega estrategias adecuadas a las necesidades del individuo, lo que exige la ampliación de los conocimientos científicos con énfasis en las ciencias del comportamiento y ecología 
humana, pues la inserción de las terapias complementarias, como el uso de las plantas medicinales, demanda mucho estudio, dedicación y responsabilidad ${ }^{8}$.

Con la finalidad de reconocer las prácticas populares y tradicionales relacionadas al consumo de plantas medicinales, fue instituido, en el año de 2008, el Programa Nacional de Plantas Medicinales y Fitoterápicos. Tal propuesta objetiva inferir, con seguridad, eficacia y calidad, las plantas medicinales, fitoterápicos y servicios correspondientes a estos, en el Sistema Único de Salud (SUS) ${ }^{4}$.

En consonancia, el Programa introdujo la Relación Nacional de Plantas Medicinales de Interés al SUS (RENISUS), compuesta por 71 plantas con potencial terapéutico. Este hecho corrobora la iniciativa de investigaciones y validación de informaciones pertinentes a la indicación y manejo de tales plantas ${ }^{4}$.

Mediante una relación social equilibrada entre el individuo y el ambiente, tenemos la posibilidad de revertir varios daños comunes a ambos, de entre los cuales destaca el uso indiscriminado de las plantas medicinales sin tener en cuenta la necesidad de la base científica para manipularlas; lo que puede inducir a la erosión de los recursos naturales, además de desencadenar toxicidades e inclusive la muerte del ser humano.

Por tanto, los profesionales de salud precisan instrumentalizarse cada día sobre el manejo adecuado de las especies vegetales en el espacio de trabajo, pues las mayoría de la población que utiliza las plantas medicinales las asocian al uso de medicamentos industrializados, sin saber que algunas mezclas terapéuticas pueden inhibir o potenciar sus efectos ${ }^{3}$.

Se sabe que para legitimar otras prácticas y conocimientos en salud, los actores sociales (médicos, enfermeras, nutricionistas, psicólogos, entre otros) necesitan de una validación intelectual, para perfeccionar las metodologías que orientarán sus acciones. Pues se percibe que la influencia del capitalismo y el acceso facilitado al consumo de remedios, todavía induce a la población a la compulsiva medicalización, siendo imprescindible evaluar el impacto de las multiterapias en las diferentes formas de cuidado, las cuales buscan reconocimiento social, importancia simbólica y refinamiento económico y tecnológico 9 .

Sobre este aspecto, conviene destacar que la pérdida de conocimiento popular sobre el uso de las plantas medicinales es evidente. Levantamientos etnobotánicos abarcan estudios sobre la diversidad vegetal en el mundo y se percibe que la concentración de tales conocimientos está restringida a la población de ancianos y a los curanderos, dada la migración de la población joven a los centros más desarrollados ${ }^{10-11}$. Sin embargo, a través del incentivo de la política nacional e investigaciones en este campo, la transmisión de las informaciones de generación a generación poco a poco está siendo rescatada.

Por lo tanto, se hace indispensable que los equipos de salud, sea el ambiente hospitalario o en espacios comunitarios, mantengan el vínculo transparente con su usuario, a fin de percibir los valores y significados referentes al cuidado con la salud que este adopte, viabilizando calidad de vida y mayor proximidad al contexto que lo rodea. Así, son rescatados, los conocimientos populares, para no desperdiciar la experiencia, además de agregar las prácticas complementarias legitimadas por la ciencia, para enriquecer el cuidado y tornarlo eficaz y seguro. 


\section{ENFERMERÍA: PERCEPCIONES Y DESAFÍOS EN EL USO DE LAS PLANTAS MEDICINALES}

En su trayecto, la ciencia ha incorporado estrategias volcadas a la calidad de la salud humana, tornándose perceptibles a los cambios graduales en los modelos de atención existentes en Brasil. De este modo, se constata la necesidad de abandonar la idea de que la enfermedad es un instrumento de trabajo de la enfermería, de manera a reorientar el enfoque a la práctica humanizada, englobando al usuario y su familia en la búsqueda por la salud.

El contexto social pasa entonces a ser valorado como fuente de informaciones relacionadas con la vida del ser humano, que muchas veces ejerce cuidados a la salud utilizando recursos presentes en el ambiente en que vive, rescatando los cuidados culturales como forma de enfrentamiento del proceso salud/enfermedad. En esta perspectiva, se percibe que el uso de las plantas medicinales es una de las prácticas de cuidado de la salud más adoptada por la población, donde el conocimiento popular continúa en la búsqueda del bienestar, sin discriminación económica y social².

Sin embargo, el conocimiento de la biodiversidad brasileña todavía presenta lagunas, y los conocimientos populares, asociados al misticismo y a la creencia, pasan a ser desconsiderados cuando el enfoque de atención a la salud da prioridad al modelo biomecanicista, hecho que evidencia la necesidad de una visión multidimensional, que abarque la intersección de las innovaciones científicas concebidas a partir de aquellas ya existentes culturalmente.

En este aspecto, la enfermería se insertae poco a poco en la adhesión de terapias complementarias a la salud, proponiendo la integridad del cuidado, práctica legalmente respaldada por la Resolución 290 de 2004 del COFEN - Consejo Federal de Enfermería, que establece y reconoce como especialidad y/o calificación del Profesional de Enfermería actuar junto a esas intervenciones ${ }^{12}$.

El enfermero se convierte en el profesional responsable para percibir los significados existentes en las diversas formas de cultura y aproximarlos a aquellos científicos, promoviendo la valoración de la biodiversidad étnica y del conocimiento empírico.

En este plano de ideas, ampliar las opciones terapéuticas a los usuarios es permitir que la naturaleza, farmacia viva, sea valorada y respetada con relación a la utilización de sus recursos naturales, los cuales pueden atender a las necesidades primarias e inclusive más específicas en salud, cuando se siguen los criterios que afectan a su manipulación correcta y valoración de la cultura del individuo como un todo, en su búsqueda de la integralidad del cuidado en salud, reafirmada en cuanto principio del $S U S^{13}$. Y en esta óptica se torna necesario trascender la estrecha relación entre el ambiente social y la eficacia del plan de cuidado destacado por el profesional de salud, lo que demanda una constante comunicación entre el ser que presta cuidados, aquel que lo recibe y la familia que lo rodea.

Se entiende, por tanto, que el SUS se constituye en un lugar privilegiado de desarrollo y expansión del uso de las plantas medicinales a favor del cuidado en salud, incorporándose como una estrategia que viene a ampliar el coeficiente de integralidad en sus prácticas en salud, asegurando la necesidad del planeamiento de las acciones en salud y la capacidad de absorber los cambios sociales como metas pluralistas, donde los profesionales de salud precisan envolverse, de modo a optimizar el cuidado, recreando formas de actuar en cada momento. Para ello, la práctica realizada por la enfermería abarca acciones con 
características, a veces, reduccionistas, con énfasis en el ser individualizado y enfermo, pero también compone prácticas que valoren la familia como espacio de diálogo, adquisición y transformación de conocimiento entre los sujetos involucrados. Y esta combinación ejercida de forma organizada y pertinente estimula el desarrollo del acto de cuidar más consciente, humanizado e integrado a la sociedad.

\section{CONSIDERACIONES FINALES}

A partir del sujeto ideológico que reflexiona, critica conceptos y se apropia de los conocimientos continuamente, se constituyen las ciencias. Y la enfermería, insertada en esta finalidad, construye su identidad, uniendo el saber racional a la subjetividad que constituye la relación social, promoviendo de esta manera la legitimidad científica de los conocimientos, entre ellos, el fomento a la investigación y el desarrollo de innovaciones tecnológicas sobre las plantas medicinales, lo que permite operar el hacer en salud, con mayor credibilidad.

Se percibe, entonces, que el ambiente pasa por un proceso constante de resistencia, dada su inmensa fuerza de establecerse en medio de la acción del ser humano, pues la exploración de su entorno contribuye al desarrollo de un sentimiento de pertenencia al medio en el cual vivimos. Y esta ideología merece ser el centro de una discusión mayor por parte de los profesionales de salud.

Visto así, se cree que la erosión del conocimiento pertinente al uso de las plantas medicinales sea uno de los factores que corroboran para la desintegración del medio, teniendo en cuenta que la sustentabilidad está siendo amenazada. Por lo tanto, urge la necesidad de comprender que nosotros también somos parte del ambiente y, por esa razón, vivimos con y para él.

Cabe a la enfermería actualizarse sobre las potencialidades de las terapias complementarias y del impacto que la cultura ejerce sobre ellas, pues se entiende que el desafío de esta profesión pasa por la valoración de las plantas medicinales en cuanto práctica del cuidado, envolviendo los recursos naturales y tradicionalmente utilizados por la mayoría de la población, como una de las estrategias para alcanzar la salud.

\section{REFERENCIAS}

1. Lorenzi H, Matos FJA. Plantas medicinais no Brasil: nativas e exóticas cultivadas. São Paulo: Instituto Plantarum, 2008.

2. Brasil Ministério da Saúde. Secretaria de ciência, tecnologia e insumos estratégicos. Política Nacional de Plantas Medicinais e Fitoterápicos. Brasília: Ministério da Saúde, 2006.

3. França ISX, Souza JÁ, Baptista RS, Britto VRS. Medicina popular: benefícios e malefícios das plantas medicinais. Rev. Bras Enferm, 2008; Brasília 61(2):201-8. [acesso em 2009 ago 05]. Disponível em: http://www.scielo.br/pdf/reben/v61n2/a09v61n2.pdf.

4. Brasil. Ministério da Saúde. Secretaria de Atenção à Saúde. Departamento de Atenção Básica. Política Nacional de Práticas Integrativas e Complementares no SUS - PNPIC-SUS / Ministério da Saúde, Secretaria de Atenção à Saúde, Departamento de Atenção Básica. Brasília: Ministério da Saúde, 2006.

5. Luz MT. Políticas de descentralização e cidadania: novas práticas em saúde no Brasil atual. In: Pinheiro R.; Mattos RA (Org). Os sentidos da integralidade na atenção e no cuidado a saúde. Rio de Janeiro. IMS - UERJ - ABRASCO; 2001. 180p.

6. Oliveira CJ, Araújo TL. Plantas medicinais: usos e crenças de idosos portadores de hipertensão arterial. Revista Eletrônica de Enfermagem [serial on line] 2007 Jan-Abr; 9(1): 
93-105. [acesso em 2009 Jun 15]. Disponível em: http://www.fen.ufg.br/revista/v9/n1/v9n1a07.htm.

7. Cunha RR, Pereira LS, Gonçalves ASR, Santos EKA, Radünz V, Heidemann ITSB. Promoção da saúde no contexto paroara: possibilidade de cuidado de enfermagem. Texto Contexto Enferm, Florianópolis, 2009 Jan-Mar; 18(1): 170-6. [acesso em 2009 nov 05]. Disponível em: http://www.scielo.br/pdf/tce/v18n1/v18n1a21.pdf.

8. Silva AL. A enfermagem na era da globalização: desafios para o século XXI. Rev LatinoAm Enfermagem, 2008; 16(4). [acesso em 2009 Jun 12]. Disponível em: http://www.scielo.br/scielo.php?script=sci arttext\&pid=S0104-11692008000400021\&lng=pt

\&nrm=iso\&tlng=pt.

9. Capra F. O ponto de mutação. Ed. Cultrix, 1992.

10. Tesser CD, Barros NF. Medicalização social e medicina alternativa e complementar: pluralização terapêutica do Sistema Único de Saúde. Rev. Saúde Pública, 2008; São Paulo 42(5):914-20. [acesso em 2009 ago 07]. Disponível em: http://www.scielo.br/pdf/rsp/v42n5/ 7115.pdf.

11. Wondimu T, Asfaw Z, Kelbessa E. Ethnobotanical study of medicinal plants around 'Dheeraa' town, Arsi Zone, Ethiopia. J Ethnopharmacol, 2007; 112(1):152-61. [acesso em 2009 ago 05]. Disponível em: http://www.ncbi.nlm.nih.gov/pubmed/17418987.

12. COFEn. Resolução 290/2004. Dispõe sobre as especialidades de enfermagem, de competência do enfermeiro. [acesso em 2009 Ago 10]. Disponível em: http://www.portalcofen.gov.br/2007/materias.asp?ArticlelD=7117\&sectionID=34.

13. Teklehaymanot T, Giday M. Ethnobotanical study of medicinal plants used by people in Zegie Peninsula, Northwestern Ethiopia. J Ethnobiol Ethnomed, 2007; 3:12. [acesso em 2009 ago 02]. Disponível em: http://www.pubmedcentral.nih.gov/articlerender.fcgi?artid=1852296. 